\title{
Identification of crucial genes in abdominal aortic aneurysm by WGCNA
}

\author{
Siliang Chen ${ }^{1}$, Dan Yang ${ }^{2}$, Chuxiang Lei ${ }^{1}$, Yuan Li ${ }^{1}$, Xiaoning Sun ${ }^{1}$, Mengyin Chen ${ }^{1}$, Xiao Wu $^{1}$, Yuehong Zheng \\ Corresp. 1 \\ ${ }^{1}$ Department of Vascular Surgery, Peking Union Medical College Hospital, Chinese Academy of Medical Sciences and Peking Union Medical College, \\ Beijing, PR China \\ 2 Department of Computational Biology and Bioinformatics, Institute of Medicinal Plant Development, Chinese Academy of Medical Sciences and Peking \\ Union Medical College, Beijing, PR China \\ Corresponding Author: Yuehong Zheng \\ Email address: yuehongzheng@yahoo.com
}

Background: Abdominal aortic aneurysm (AAA) is the full thickness dilation of the abdominal aorta. However, few effective medical therapies are available. Thus, elucidating the molecular mechanism of AAA pathogenesis and exploring the potential molecular target of medical therapies for AAA is of vital importance.

Methods: Three expression datasets (GSE7084, GSE47472 and GSE57691) were downloaded from the Gene Expression Omnibus (GEO). These datasets were merged and then normalized using the "sva" $\mathrm{R}$ package. Differential expressed gene (DEG) analysis and weighted gene co-expression network analysis (WGCNA) were conducted. We compared the co-expression patterns between AAA and normal conditions, and hub genes of each functional module were identified. DEGs were mapped to co-expression network under AAA condition and a DEG co-expression network was generated. Crucial genes were identified using MCODE (a plugin in Cytoscape).

Results: In our study, 6 and 10 gene modules were detected for the AAA and normal conditions, respectively, while 143 DEGs were screened. Compared to the normal condition, genes associated with immune response, inflammation and muscle contraction were clustered in 3 gene modules respectively under the AAA condition; the hub genes of the 3 modules were MAP4K1, NFIB and HPK1, respectively. A DEG co-expression network with 102 nodes and 303 edges was identified, and a hub gene cluster with 10 genes from the DEG co-expression network was detected. YIPF6, RABGAP1, ANKRD6, GPD1L, PGRMC2, HIGD1A, GMDS, MGP, SLC25A4 and FAM129A were in the cluster. The expression levels of these 10 genes showed potential diagnostic value.

Conclusion: Based on WGCNA, we detected 6 modules under the AAA condition and 10 modules in the normal condition. Hub genes of each module and hub gene clusters of the DEG co-expression network were identified. These genes may act as potential targets for medical therapy and diagnostic biomarkers. Further studies are needed to elucidate the detailed biological function of these genes in the pathogenesis of AAA. 


\section{Identification of Crucial Genes in Abdominal Aortic Aneurysm by 2 WGCNA}

3

4 Siliang Chen ${ }^{1}$, Dan Yang ${ }^{2}$, Chuxiang Lei ${ }^{1}$, Yuan $\mathrm{Li}^{1}$, Xiaoning Sun ${ }^{1}$, Mengyin Chen ${ }^{1}$, Xiao $\mathrm{Wu}^{1}$, 5 Yuehong Zheng ${ }^{1 \#}$

6 'Department of Vascular Surgery, Peking Union Medical College Hospital, Chinese Academy of

7 Medical Sciences and Peking Union Medical College, Beijing, PR China

$8{ }^{2}$ Department of Computational Biology and Bioinformatics, Institute of Medicinal Plant

9 Development, Chinese Academy of Medical Sciences and Peking Union Medical College, Beijing, 10 PR China

11 Corresponding Author:

12 Yuehong Zheng ${ }^{1}$

13 No. 1 Shuai Fu Yuan, Dongcheng District, Beijing, 100730, China

14 Email address: yuehongzheng@yahoo.com 
38

39

40

41

42

43

44

45

46

47

48

49

50

51

52

53

54

55

56

57

58

59

60

61

62

63

64

65

66

67

68

69

70

71

72

73

74

75

76

\section{Abstract}

Background: Abdominal aortic aneurysm (AAA) is the full thickness dilation of the abdominal aorta. However, few effective medical therapies are available. Thus, elucidating the molecular mechanism of AAA pathogenesis and exploring the potential molecular target of medical therapies for AAA is of vital importance.

Methods: Three expression datasets (GSE7084, GSE47472 and GSE57691) were downloaded from the Gene Expression Omnibus (GEO). These datasets were merged and then normalized using the "sva" R package. Differential expressed gene (DEG) analysis and weighted gene coexpression network analysis (WGCNA) were conducted. We compared the co-expression patterns between AAA and normal conditions, and hub genes of each functional module were identified. DEGs were mapped to co-expression network under AAA condition and a DEG co-expression network was generated. Crucial genes were identified using MCODE (a plugin in Cytoscape).

Results: In our study, 6 and 10 gene modules were detected for the AAA and normal conditions, respectively, while 143 DEGs were screened. Compared to the normal condition, genes associated with immune response, inflammation and muscle contraction were clustered in 3 gene modules respectively under the AAA condition; the hub genes of the 3 modules were MAP4K1, NFIB and HPK1, respectively. A DEG co-expression network with 102 nodes and 303 edges was identified, and a hub gene cluster with 10 genes from the DEG co-expression network was detected. YIPF6, RABGAP1, ANKRD6, GPD1L, PGRMC2, HIGD1A, GMDS, MGP, SLC25A4 and FAM129A were in the cluster. The expression levels of these 10 genes showed potential diagnostic value.

Conclusion: Based on WGCNA, we detected 6 modules under the AAA condition and 10 modules in the normal condition. Hub genes of each module and hub gene clusters of the DEG coexpression network were identified. These genes may act as potential targets for medical therapy and diagnostic biomarkers. Further studies are needed to elucidate the detailed biological function of these genes in the pathogenesis of AAA.

\section{Introduction}

Abdominal aortic aneurysm (AAA) is a type of true aneurysm located at the abdominal aorta, and it is defined as permanent and irreversible dilation of the abdominal aorta to $50 \%$ more than the normal aortic diameter (Sakalihasan et al., 2018). The prevalence of AAA in populations above 65 years old is approximately $4 \% \sim 8 \%$, and it is the main cause of death in the senior population. Furthermore, the mortality of a ruptured AAA is almost $100 \%$ if left untreated (Nordon, Hinchliffe, Loftus, \& Thompson, 2011; Sakalihasan et al., 2018). Open surgical repair and endovascular repair remain two main methods to treat AAA. However, there are temporarily few effective medical therapies to treat AAA (Golledge, 2019). Therefore, elucidating the molecular mechanism of AAA pathogenesis and exploring the potential molecular targets of medical therapies for AAA is of vital importance. Although the molecular mechanism of AAA pathogenesis is not yet clear, it may involve biological processes such as immune response, chronic inflammation, oxidative stress, phenotypic switching of smooth muscle, and degradation of extracellular matrix, etc. (Golledge,

Peer] reviewing PDF | (2019:05:37692:2:0:NEW 5 Sep 2019) 
Muller, Daugherty, \& Norman, 2006; Raffort et al., 2017; Weintraub, 2009). Abundant studies have been performed on the molecular mechanism of AAA pathogenesis(Golledge, 2019; Raffort et al., 2017; Sakalihasan et al., 2018), but weighted gene co-expression network analysis (WGCNA) has not been used to construct a gene co-expression network in AAA.

WGCNA was developed by Horvath and Zhang in 2005 (Langfelder \& Horvath, 2008; B. Zhang \& Horvath, 2005), and an R package is available on the official website of $\mathrm{R}$ (https://cran.rproject.org/). WGCNA can be used to construct a weighted gene co-expression network, detect gene modules, correlate gene modules with clinical traits and identify intramodular hub genes (Langfelder \& Horvath, 2008; van Dam, Vosa, van der Graaf, Franke, \& de Magalhaes, 2018; B. Zhang \& Horvath, 2005). Furthermore, WGCNA can be used not only to construct co-expression networks based on coding RNA expression data but also to construct noncoding RNA coexpression networks, such as miRNA co-expression networks and IncRNA co-expression networks (Hu et al., 2019; Ma et al., 2019).

Previous studies using microarray data of AAA have conducted differentially expressed gene (DEG) analysis and functional enrichment analysis. Gene expression profiles were compared between AAA and normal abdominal aorta, and validated genes such as CTLA4, NKTR and CD8A were identified in DEG analysis (Biros et al., 2015; Lenk et al., 2007). Nevertheless, DEGs and functional enrichment analysis cannot reveal connections and interactions among genes that are crucial in biological processes.

In our study, 3 GEO mRNA microarray datasets (Table 1) were used to conduct WGCNA. Gene co-expression networks and gene modules were constructed in both AAA expression data and normal expression data. Due to the lack of clinical trait data, we used genes in all gene modules to conduct functional enrichment analysis and then mapped the DEGs to whole gene expression networks of both the AAA and normal expression data. Furthermore, we identified 10 hub genes and showed their diagnostic values by receiver operating characteristic analysis.

\section{Materials \& Methods}

\section{Datasets}

The Gene Expression Omnibus (GEO) database (http://www.ncbi.nlm.nih.gov/geo/) was searched with the keywords "abdominal aortic aneurysm" [Title] AND ("Series" [Entry type] AND "Homo sapiens"[Organism]). Finally, 3 datasets in the search results were included in our study: GSE7084, GSE47472 and GSE57691. Series matrix files and data tables of the microarray platform were downloaded from the GEO website.

\section{DEG analysis}

The 3 series matrix files were annotated with an official gene symbol using the data table of the microarray platform, and then gene expression matrix files were obtained. The 3 gene expression matrix files were merged into one file, and the "sva" $\mathrm{R}$ package was used to conduct batch normalization of the expression data from the three different datasets. Finally, a normalized gene expression matrix file containing data from the three different datasets was obtained for 
117 differentially expressed gene (DEG) analysis. The "limma" R package was used to conduct DEG

118

119

120

121

122

123

124

125

126

127

128

129

130

131

132

133

134

135

136

137

138

139

140

141

142

143

144

145

146

147

148

149

150

151

152

153

154

155

156

analysis. The threshold of DEGs was set as $\mid \log _{2}$ (fold-change) $\mid>0.8$ and $p<0.05$.

\section{Co-expression network construction by WGCNA}

Principal component analysis (PCA) was conducted using the whole gene list and DEG list. The 2D-PCA plots showed that the variation between the AAA and normal groups was not significant ( Figure S1). Therefore, conducting WGCNA analysis using samples from AAA and normal groups separately was reasonable. Then, the "WGCNA" R package was used to construct a coexpression network for all genes in AAA and normal abdominal aorta samples. Genes with the top $25 \%$ variance were filtered by the algorithm for further analysis. Then 70 AAA samples were involved in one WGCNA analysis, while 26 normal samples were involved in another WGCNA analysis. Samples were used to calculate the Pearson's correlation matrices. Then, the weighted adjacency matrix was created with the formula $a_{m n}=\left|c_{m n}\right|^{\beta}$ (where $\mathrm{a}_{\mathrm{mn}}$ : adjacency between gene $\mathrm{m}$ and gene $\mathrm{n}, \mathrm{c}_{\mathrm{mn}}$ : Pearson's correlation, and $\beta$ : soft-power threshold). Furthermore, the weighted adjacency matrix was transformed into a topological overlap measure (TOM) matrix to estimate its connectivity property in the network. Average linkage hierarchical clustering was used to construct a clustering dendrogram of the TOM matrix. The minimal gene module size was set to 30 to obtain appropriate modules, and the threshold to merge similar modules was set to 0.25 .

\section{Functional and pathway enrichment of gene modules}

To obtain the biological functions and signaling pathways involved in each module, genes in each module were subjected to gene ontology (GO) analysis and Kyoto Encyclopedia of Genes Genomes (KEGG) pathway analysis using The Database for Annotation, Visualization and Integrated Discovery (DAVID) v6.8 (ttps://david.ncifcrf.gov/). The threshold was set as count $>2$ and $\mathrm{p}<0.05$. To compare the two networks based on modules, the percentage of related genes in the important pathways of the modules we interested in under the AAA condition and corresponding modules under the normal condition were calculated. Chi-square tests were used to compare the percentages.

\section{Identification of the hub genes in functional modules and crucial gene mining}

Each gene with the highest intramodular connectivity in each functional module calculated by the WGCNA algorithm was identified as a hub gene. Then, DEGs were mapped to the whole coexpression network of AAA and normal samples using Cytoscape v3.7.0, and DEG co-expression networks were obtained. The DEG co-expression network of AAA was analyzed by Molecular Complex Detection (MCODE), a plugin in Cytoscape that clusters a given network based on topology to find densely connected regions, and the most significant cluster with 10 nodes was visualized. Then, receiver operating characteristic (ROC) analysis was conducted with 10 genes in the cluster using SPSS 25.0.

\section{Results}

Peer] reviewing PDF | (2019:05:37692:2:0:NEW 5 Sep 2019) 
157

158

159

160

161

162

163

164

165

166

167

168

169

170

171

172

173

174

175

176

177

178

179

180

181

182

183

184

185

186

187

188

189

190

191

192

193

194

195

\section{Workflow}

The workflow is shown in Figure 1. DEG identification was conducted, and 143 DEGs were screened. WGCNA on the AAA and normal samples was performed. Co-expression networks for the AAA and normal conditions were constructed, and gene modules were also detected for further hub gene identification and comparison of expression patterns between the AAA and normal samples. Then, the DEGs were mapped to the AAA co-expression network and a DEG coexpression network was obtained. Crucial genes were identified based on this network, and ROC analysis was conducted using these crucial genes.

\section{Identification of DEGs}

A total of 143 DEGs were identified with the threshold at $\mid \log _{2}$ (fold-change) $\mid>0.8$ and $p<0.05$ and consisted of 125 down-regulated genes and 18 up-regulated genes. The expression patterns of the DEGs are shown in Figure 2. Down-regulated genes and up-regulated genes with a top 10-fold change ratio are shown in Table S1.

\section{Construction of the co-expression network of the AAA and normal conditions}

The sample clustering dendrograms of the AAA and normal conditions are shown in Figures $3 \mathrm{~A}$ and $4 \mathrm{~A}$, respectively. In both conditions, all samples were included in the clusters. The softpower threshold $\beta$ was determined by the function "sft\$powerEstimate"; $\beta=5$ and $\beta=16$ were selected for further analysis of the AAA and normal conditions, respectively (figures $3 \mathrm{~B}, 3 \mathrm{C}, 3 \mathrm{D}$, $3 \mathrm{E}$ andfigures S2B, S2C, S2D, S2E). Then, gene modules were detected based on the TOM matrix. In the analysis, 6 modules were detected under the AAA condition, while 10 modules were detected under the normal condition (figures 3F, 3G and figures S2F, S2G).

\section{Comparison of the co-expression patterns between the AAA and normal conditions}

Next, we compared the co-expression patterns between the AAA and normal conditions by examining their functional modules. We investigated the biological function of the genes in each module. GO-BP and KEGG pathway analyses were conducted. The results of the GO-BP and KEGG pathway analyses for each module are shown in Table S2 \& Table S3. In the network analysis, 3 modules in the AAA condition may be associated with the pathogenesis of AAA, and are shown in black, red and turquoise. The GO-BP terms and KEGG pathways with the top 10 counts of the 3 modules are shown in Figure 4, Table S4 \& Table S5. The black module is mainly associated with the immune response and T/B cell activation, while the turquoise module is mainly associated with the inflammatory response and signaling pathways in inflammation. The red module is mainly associated with muscle contraction and focal adhesion. GO-CC and GO-MF analyses were also conducted (data not shown).

In the normal condition, GO-BP terms and KEGG pathways were associated with immune response and inflammatory response in some of the modules. However, these terms and pathways were scattered in each module and are not enriched in one or two modules. Furthermore, GO-BP 
196

197

198

199

200

201

202

203

204

205

206

207

208

209

210

211

212

213

214

215

216

217

218

219

220

221

222

223

224

225

226

227

228

229

230

231

232

233

234

235

terms and KEGG pathways associated with muscle contraction and focal adhesion were even more scattered in each module.

Then, we compared the co-expression pattern of the two networks computationally and systematically based on the results of the KEGG pathway analysis of the gene modules. Using the black module in AAA as an example, we calculated the percentage of genes associated with immune response (hematopoietic cell lineage, primary immunodeficiency, B cell receptor signaling pathway, $\mathrm{T}$ cell receptor signaling pathway, intestinal immune network for IgA production, and natural killer cell mediated cytotoxicity) in all genes of the KEGG pathways with the top 10 counts $\left(\frac{\text { Related genes }}{\text { All genes }} * 100 \%\right.$, where Related genes $=$ the number of genes associated with targeted pathways in KEGG pathways with the top 10 counts, and All genes= the number of all genes in KEGG pathways with the top 10 counts). We found that the pathways associated with the immune response were mainly distributed in the dark green module in the normal condition and calculated the percentage of genes associated with the immune response in all genes of the KEGG pathways with the top 10 counts $\left(\frac{\text { Related genes }}{\text { All genes }} * 100 \%\right)$. Next, we compared the two percentages using the chi-square test, and the percentage in the black module in the AAA condition was significantly larger than the percentage in the dark green module in the normal condition $(\mathrm{p}<0.05)$. A similar analysis was conducted on the red and turquoise modules for the AAA condition and the corresponding modules for the normal condition. We also explained these variables in detail (Data $\mathrm{S} 1$ ). The results are shown in Table 2. These results showed that the pathways associated with inflammation, immune response, and vascular smooth muscle contraction were scattered in the normal condition.

Taken together, the immune response, inflammation and vascular smooth muscle contraction play an important role in the pathogenesis of AAA.

\section{Identification of hub genes in the functional modules}

Intramodular connectivity was calculated by the WGCNA algorithm. Genes with the highest intramodular connectivity were selected as hub genes in each module in both the AAA and normal conditions (Table S6).

The hub genes of the black, turquoise and red modules in the AAA condition were MAP4K1, STYX and NFIB, respectively. MAP4K1 is a regulator of JNK, immune cell adhesion and T and $\mathrm{B}$ cell activation (Chuang, Wang, \& Tan, 2016) and is also associated with T, B cell activation andcytokine production (Alzabin, Bhardwaj, Kiefer, Sawasdikosol, \& Burakoff, 2009; Shui et al., 2007). STYX regulates apoptosis through cross-talk with FBXW7 (Reiterer, Figueras-Puig, et al., 2017). NFIB is a nuclear transcription factor, and its detailed function is still not known, but it may be associated with an immune system threshold (Liston, Papadopoulou, Danso-Abeam, \& Dooley, 2012).

\section{Mining crucial genes mediating AAA}

DEGs were mapped to the whole co-expression network in the AAA condition using Cytoscape, and a DEG co-expression network was obtained. The threshold of weighted edges was set as 0.2 
236

237

238

239

240

241

242

243

244

245

246

247

248

249

250

251

252

253

254

255

256

257

258

259

260

261

262

263

264

265

266

267

268

269

270

271

272

273

274

275

and 102 nodes, and 303 edges are in the DEG co-expression network (Figure 5A). Then, GO-BP and KEGG analyses were conducted using genes from the DEG co-expression network, and the results are shown in Table S7. These genes were mainly associated with oxidative stress and inflammation. The most significant cluster of the DEG co-expression network detected by MCODE consisted of 10 genes and was visualized (Figure 5B Table 3). Yip1 domain family member 6 (YIPF6), a protein associated with clathrin-derived vesicle budding and vesiclemediated transport, was the gene with the highest degree in the cluster.

All 10 genes were selected for ROC analysis. The area under the curve (AUC) of each gene is listed in Table 4. HIGD1A (HIG1 hypoxia inducible domain family member 1A) has the highest AUC value ( $\mathrm{AUC}=0.886, \mathrm{SE}=0.037, \mathrm{p}<0.001$ ), while MGP (matrix Gla protein) has the lowest AUC value $(\mathrm{AUC}=0.742, \mathrm{SE}=0.050, \mathrm{p}<0.001)$. The ROC curves of the genes with AUC exceeding 0.8 were plotted (Figure 6). These genes were YIPF6, PGRMC2, ANKRD46, GPD1L, HIGD1A, GMDS, FAM129A and SLC25A4. Thus, these genes have potential diagnostic value and may become biomarkers for AAA; however, they need to be further validated in future studies.

\section{Discussion}

In our present study, 143 DEGs were screened (Figure 2, Table S1). WGCNA was used to construct a co-expression network and detect gene modules in both AAA and normal conditions. In our analysis, 6 modules in the AAA condition and 10 modules in the normal condition were detected based on the co-expression network. The gene modules were subjected to GO-BP and KEGG pathway analyses, and co-expression patterns between the AAA and normal conditions were compared (Figure 4 and Table S2, S3). In the AAA condition, the black module was mainly associated with the immune response and T/B cell activation, while the turquoise module was mainly associated with the inflammatory response and signaling pathways in inflammation. The red module was mainly associated with muscle contraction and focal adhesion. In the normal condition, the GO-BP terms and KEGG pathways associated with immune response, inflammatory response, muscle contraction and focal adhesion were scattered in each module and were not enriched in one or two modules. These differences suggest that the immune response, inflammation and vascular smooth muscle play important roles in the pathogenesis of AAA. For each module, the genes with the highest intramodular connectivity were selected as the hub genes (Table S6). Then, DEGs were mapped to the co-expression network of AAA, and a DEG coexpression network with 102 nodes and 303 edges was obtained (Figure 5A). The Hub gene cluster was the most significant cluster in the DEG co-expression network selected by MCODE. Ten genes were in the cluster: YIPF6, RABGAP1, ANKRD46, GPD1L, PGRMC2, HIGD1A, GMDS, MGP, FAM129A, and SLC25A4 (Figure 5B). These genes are potentially crucial genes in the pathogenesis of AAA and showed diagnostic value (Figure 6).

A previous study using the same GEO datasets that we used screened 1199 DEGs (Wan, Huang, $N i, \& Y u, 2018)$, which is far more than the number of DEGs we screened. However, unlike the previous study, we used $\log _{2} \mid$ FoldChange $\mid>0.8$ as another threshold, and the adjusted $p$ value was used. Furthermore, the "sva" R package was used to normalize the 3 datasets before DEG analysis

PeerJ reviewing PDF | (2019:05:37692:2:0:NEW 5 Sep 2019) 
276 in our study. The differences in choosing parameters and normalization may account for the 277 different number of DEGs screened.

278 Previous genome-wide association studies (GWASs) reported some significant AAA-associated 279 SNPs and genes from genomic data. Helgadottir et al. discovered one common variant 280 (rs10757278-G on chromosome 9p21, OR=1.31, $\mathrm{P}=1.2^{*} 10^{-12}$ ) that is associated with the risk of 281 AAA (Helgadottir et al., 2008). Other SNPs, such as rs7025486 on 9q33 in the DAB2IP gene 282 ( $\mathrm{OR}=1.21$ and $\left.\mathrm{P}=4.6^{*} 10^{-10}\right)$ (Gretarsdottir et al., 2010), rs1466535 on 12q13.3 in the LRP1 gene $283\left(\mathrm{OR}=1.15\right.$ and $\left.\mathrm{P}=, 4.52^{*} 10^{-10}\right)$ (Bown et al., 2011), rs599839 on 1p13.3 in the SORT1 gene $284\left(\mathrm{OR}=0.81\right.$ and $\left.\mathrm{P}=7.2^{*} 10^{-14}\right)$ (Jones et al., 2013) and rs6511720 in LDLR (OR=0.76, $\left.\mathrm{p}=2.08^{*} 10^{-10}\right)$ 285 (Bradley et al., 2013), are significantly associated with AAA. Jones et al. conducted a meta286 analysis of GWAS for AAA and identified 1q32.3 (SMYD2), 13q12.11 (LINC00540), 20q13.12 287 (near PCIF1/MMP9/ZNF335), and 21q22.2 (ERG) as four new loci associated with AAA and 288 found that these four loci may be specifically associated with AAA compared with other 289

290

291

292

293

294

295

296

297

298

299

300

301

302

303

304

305

306

307

308

309

310

311

312

313

314

315 cardiovascular diseases (Jones et al., 2017). We screened DEGs based on transcriptomic data, and there was some overlap between previous GWASs and DEGs we screened. MMP9 is in the DEG list with a fold-change of 2.03, which is in accord with the study by Jones et al., which found that one SNP near PCIF1/MMP9/ZNF335 was significantly associated with AAA (Jones et al., 2017). Furthermore, one SNP in LRP1 was associated with AAA, and Boucher et al. found that the LRP1 gene plays a protective role in vascular disease via TGF- $\beta$ signaling (Boucher et al., 2007; Bown et al., 2011). Thus, the TGFBR3 gene with a fold-change of -1.01 in our DEG list may be associated with LRP1 gene function and pathogenesis of AAA, although the detailed mechanism has not been clearly elucidated. Furthermore, we obtained genes closest to SNPs in those literature respectively (i.e. GWAS genes)(Table S8) using dbSNP (https://www.ncbi.nlm.nih.gov/snp/) and found that there were 2 GWAS genes (GSN and MMP9) overlapped with DEGs. Then we compared the GWAS genes with genes enriched in GO-BP and KEGG pathway terms of DEGs in the co-expression network (Table S7) and there were also no overlap. Therefore, we inferred that GWAS genes were not "close" to DEGs in the co-expression network in the functional level. We also evaluated the association between SNPs and DEGs using eQTL calculator in GTEx website (https://gtexportal.org/home/testyourown)(Brown et al., 2017). Although some NES (normalized effect size) between some SNPs and genes were significant (Table S9), the biological significance is not clear and further validation is needed. Some researchers combined genomic data and patient history of AAA. Li et al. integrated genomic data of AAA based on whole genome sequencing and electronic health record data using a machine learning approach. Their study provided a paradigm for both understanding the pathogenesis of complex diseases such as AAA and managing the personal health of patients (Li et al., 2018). Duren et.al developed a novel method (PECA, paired expression chromatin accessibility) to construct enhancer gene network and enable us to view how the regulatory elements works (Duren, Chen, Jiang, Wang, \& Wong, 2017). Both of these studies provided future directions for studying the pathogenesis of AAA.

The comparison of gene modules based on GO-BP and KEGG between the AAA and normal conditions indicates that these two conditions have different co-expression patterns. Using RNA 
316 microarray, Spin et al. showed that in AAA mice, immune genes were widely upregulated (Spin 317 et al., 2011). Furthermore, evidence for the presence of innate and adaptive immune systems in 318 the pathogenesis of AAA has been provided by many studies (Coscas et al., 2018; Lareyre et al., 319 2017; Senemaud et al., 2017; Trachet et al., 2016). Our finding that the immune response was

320

321

322

323

324

325

326

327

328

329

330

331

332

333

334

335

336

337

338

339

340

341

342

343

344

345

346

347

348

349

350

351

352

353

354

355 clustered in the black module is consistent with the studies mentioned above. Inflammation also plays an important role in the pathogenesis of AAA. In addition, Spin et al. found that inflammatory genes are also up-regulated in AAA mice (Spin et al., 2011). Cells related to inflammation such as B cells, T cells, natural killer cells and macrophages and their products have been found in AAA tissue (Biros et al., 2014; Furusho et al., 2018; Meng et al., 2016; Tsuruda et al., 2008; J. Zhang et al., 2011). Studies have also shown that regulatory T cells can inhibit AAA development in human and animal models such as mice by inhibiting the inflammatory response in AAA (Ait-Oufella et al., 2013; Yodoi et al., 2015; Zhou et al., 2015), which further supports the inflammation-mediated theory of AAA. Our findings on the turquoise module agree with the results reported by these studies. Our study has also shown that vascular smooth muscle contraction is associated with AAA pathogenesis. Unlike the immune response and inflammation, little is known about the relationship between vascular smooth muscle contraction and the pathogenesis of AAA. However, Chew et al. found that elastase and matrix metalloproteinase (MMP, mainly MMP2 and MMP9) both promote early dilation of AAA by inhibiting vascular smooth muscle contraction through the mechanism of $\mathrm{Ca}^{2+}$ entry inhibition of vascular smooth muscle (Chew, Conte, \& Khalil, 2004; Chew, Orshal, \& Khalil, 2003, 2004). Based on the GOBP and KEGG pathway analyses, we found that the genes in the DEG co-expression network were mainly associated with oxidative stress and inflammation. Emeto et al. summarized that inflammation, oxidative stress and reactive oxygen species play an important role in the pathogenesis of AAA and believed that oxidative stress induces inflammation and that tissue injury is caused in the pathogenesis of AAA (Emeto, Moxon, Au, \& Golledge, 2016).

The hub genes of the black, turquoise and red modules in the AAA condition were mitogenactivated protein kinase kinase kinase kinase 1 (MAP4K1), serine/threonine/tyrosine interacting protein (STYX) and nuclear factor I B (NFIB), respectively. MAP4K1, also named hematopoietic progenitor kinase 1 (HPK1), is expressed in T cells, B cells, and macrophages, as well as hematopoietic progenitor cells. It serves as a regulator of JNK and $\mathrm{T}$ and $\mathrm{B}$ cell signaling. It can also regulate immune cell adhesion (Chuang et al., 2016) and is associated with $\mathrm{T}$ and $\mathrm{B}$ cell activation and cytokine production (Alzabin et al., 2009; Shui et al., 2007), which is consistent with our findings in the functional enrichment analysis of the black module in the AAA condition. STYX is a pseudophosphatase with no actual catalytic activity. It serves as an anchor for MAPKs and plays an important role in their function (Reiterer, Pawlowski, \& Farhan, 2017). In addition, STYX regulates apoptosis in breast cancer cells and HeLa cells through cross-talk with FBXW7 (Reiterer, Figueras-Puig, et al., 2017). Therefore, STYX may also play a role in regulating vascular smooth muscle apoptosis in the pathogenesis of AAA. NFIB is a nuclear transcription factor; however, its detailed functions are still not known (Ono \& Okada, 2018). It can be downregulated by microRNA-29, which is an immune system threshold. In conclusion, these 3 
356 hub genes from the 3 key WGCNA gene modules may be of vital importance in the pathogenesis 357 of AAA.

358 YIPF6 was the gene with the highest degree in the hub gene cluster generated from the DEG

359

360

361

362

363

364

365

366

367

368

369

370

371

372

373

374

375

376

377

378

379

380

381

382

383

384

385

386

387

388

389

390

391

392

393

394

395

396

397

398

co-expression network. YIPF6 is a member of the Yip1 family of proteins and is located in the Golgi apparatus. It is believed to participate in vesicular transport (Kranjc et al., 2017). Brandl et al. discovered that one type of null mutation of YIPF6 induces spontaneous intestinal inflammation in mice, but the mechanism seems to be related to granule secretion by Paneth and goblet cells (Brandl et al., 2012). Whether YIPF6 is associated with chronic inflammation in AAA is unknown, but it may play a role in the balance between proinflammatory and anti-inflammatory cytokine production. However, the ROC curve showed that YIPF6 as well as other genes in this cluster has potential diagnostic value.

In this study, we used WGCNA to construct a co-expression network, detect gene modules and identify hub genes in AAA for the first time. In addition, we merged and normalized 3 GEO datasets instead of using only one to conduct our analysis. However, due to the lack of clinical trait data in the 3 GEO datasets, we did not correlate gene modules with clinical traits when conducting the WGCNA.

Here, our study revealed that the co-expression patterns between the AAA and normal conditions are different. These differences indicate that immune response, inflammation and vascular smooth muscle contraction may play important roles in the pathogenesis of AAA. Then, we identified hub genes of gene modules in both the AAA and normal conditions and hub gene clusters of the DEG co-expression network. These crucial genes may have crucial biological functions in the pathogenesis of AAA.

\section{Conclusions}

Based on WGCNA, we detected 6 modules in the AAA condition and 10 modules in the normal condition. Hub genes of each module and hub gene clusters of the DEG co-expression network were identified. These genes may act as potential targets for medical therapy and diagnostic biomarkers. Further studies are needed to elucidate the detailed biological function of these genes in the pathogenesis of AAA.

\section{Acknowledgments}

This work was supported by grants from the Natural Science Foundation of China (81770481 and 51890894), Natural Science Foundation of Beijing (7172171), CAMS Innovation Fund for Medical Sciences (CIFMS, 2017-I2M-1-008).

\section{References}

Ait-Oufella, H., Wang, Y., Herbin, O., Bourcier, S., Potteaux, S., Joffre, J., . . Mallat, Z. (2013). Natural regulatory T cells limit angiotensin II-induced aneurysm formation and rupture in mice. Arterioscler Thromb Vasc Biol, 33(10), 2374-2379. doi:10.1161/atvbaha.113.301280

Alzabin, S., Bhardwaj, N., Kiefer, F., Sawasdikosol, S., \& Burakoff, S. (2009). Hematopoietic progenitor kinase 1 is a negative regulator of dendritic cell activation. $J$ Immunol, 182(10), 6187-6194. doi:10.4049/jimmunol.0802631

Biros, E., Gabel, G., Moran, C. S., Schreurs, C., Lindeman, J. H., Walker, P. J., . . Golledge, J. (2015). Differential gene expression in human abdominal aortic aneurysm and aortic occlusive disease. Oncotarget, 6(15), 12984-

Peer] reviewing PDF | (2019:05:37692:2:0:NEW 5 Sep 2019) 
12996. doi:10.18632/oncotarget.3848

Biros, E., Moran, C. S., Rush, C. M., Gabel, G., Schreurs, C., Lindeman, J. H., . . Golledge, J. (2014). Differential gene expression in the proximal neck of human abdominal aortic aneurysm. Atherosclerosis, 233(1), 211218. doi:10.1016/j.atherosclerosis.2013.12.017

Boucher, P., Li, W. P., Matz, R. L., Takayama, Y., Auwerx, J., Anderson, R. G., \& Herz, J. (2007). LRP1 functions as an atheroprotective integrator of TGFbeta and PDFG signals in the vascular wall: implications for Marfan syndrome. PLoS One, 2(5), e448. doi:10.1371/journal.pone.0000448

Bown, M. J., Jones, G. T., Harrison, S. C., Wright, B. J., Bumpstead, S., Baas, A. F., . . Samani, N. J. (2011). Abdominal aortic aneurysm is associated with a variant in low-density lipoprotein receptor-related protein 1. Am J Hum Genet, 89(5), 619-627. doi:10.1016/j.ajhg.2011.10.002

Bradley, D. T., Hughes, A. E., Badger, S. A., Jones, G. T., Harrison, S. C., Wright, B. J., , . Bown, M. J. (2013). A variant in LDLR is associated with abdominal aortic aneurysm. Circ Cardiovasc Genet, 6(5), 498-504. doi:10.1161/circgenetics.113.000165

Brandl, K., Tomisato, W., Li, X., Neppl, C., Pirie, E., Falk, W., . . Beutler, B. (2012). Yip1 domain family, member 6 (Yipf6) mutation induces spontaneous intestinal inflammation in mice. Proc Natl Acad Sci U S A, 109(31), 12650-12655. doi:10.1073/pnas.1210366109

Brown, A. A., Vinuela, A., Delaneau, O., Spector, T. D., Small, K. S., \& Dermitzakis, E. T. (2017). Predicting causal variants affecting expression by using whole-genome sequencing and RNA-seq from multiple human tissues. Nat Genet, 49(12), 1747-1751. doi:10.1038/ng.3979

Chew, D. K., Conte, M. S., \& Khalil, R. A. (2004). Matrix metalloproteinase-specific inhibition of Ca2+ entry mechanisms of vascular contraction. J Vasc Surg, 40(5), 1001-1010. doi:10.1016/j.jvs.2004.08.035

Chew, D. K., Orshal, J. M., \& Khalil, R. A. (2003). Elastase-induced suppression of endothelin-mediated Ca2+ entry mechanisms of vascular contraction. Hypertension, 42(4), 818-824. doi:10.1161/01.Hyp.0000086200.93184.8e

Chew, D. K., Orshal, J. M., \& Khalil, R. A. (2004). Elastase promotes aortic dilation by inhibiting Ca2+ influx into vascular smooth muscle. J Cardiovasc Pharmacol, 43(4), 504-513.

Chuang, H. C., Wang, X., \& Tan, T. H. (2016). MAP4K Family Kinases in Immunity and Inflammation. Adv Immunol, 129, 277-314. doi:10.1016/bs.ai.2015.09.006

Coscas, R., Dupont, S., Mussot, S., Louedec, L., Etienne, H., Morvan, M., . . . Michel, J. B. (2018). Exploring antibody-dependent adaptive immunity against aortic extracellular matrix components in experimental aortic aneurysms. J Vasc Surg, 68(6s), 60S-71S.e63. doi:10.1016/j.jvs.2017.11.090

Duren, Z., Chen, X., Jiang, R., Wang, Y., \& Wong, W. H. (2017). Modeling gene regulation from paired expression and chromatin accessibility data. Proc Natl Acad Sci U S A, 114(25), E4914-e4923. doi:10.1073/pnas. 1704553114

Emeto, T. I., Moxon, J. V., Au, M., \& Golledge, J. (2016). Oxidative stress and abdominal aortic aneurysm: potential treatment targets. Clin Sci (Lond), 130(5), 301-315. doi:10.1042/cs20150547

Furusho, A., Aoki, H., Ohno-Urabe, S., Nishihara, M., Hirakata, S., Nishida, N., . . . Fukumoto, Y. (2018). Involvement of B Cells, Immunoglobulins, and Syk in the Pathogenesis of Abdominal Aortic Aneurysm. $J$ Am Heart Assoc, 7(6). doi:10.1161/jaha.117.007750

Golledge, J. (2019). Abdominal aortic aneurysm: update on pathogenesis and medical treatments. Nat Rev Cardiol, 16(4), 225-242. doi:10.1038/s41569-018-0114-9

Golledge, J., Muller, J., Daugherty, A., \& Norman, P. (2006). Abdominal aortic aneurysm: pathogenesis and implications for management. Arterioscler Thromb Vasc Biol, 26(12), 2605-2613. doi:10.1161/01.ATV.0000245819.32762.cb

Gretarsdottir, S., Baas, A. F., Thorleifsson, G., Holm, H., den Heijer, M., de Vries, J. P., . . S Stefansson, K. (2010). Genome-wide association study identifies a sequence variant within the DAB2IP gene conferring susceptibility to abdominal aortic aneurysm. Nat Genet, 42(8), 692-697. doi:10.1038/ng.622

Helgadottir, A., Thorleifsson, G., Magnusson, K. P., Gretarsdottir, S., Steinthorsdottir, V., Manolescu, A., . . . Stefansson, K. (2008). The same sequence variant on 9p21 associates with myocardial infarction, abdominal aortic aneurysm and intracranial aneurysm. Nat Genet, 40(2), 217-224. doi:10.1038/ng.72

Hu, Z., Yang, D., Tang, Y., Zhang, X., Wei, Z., Fu, H., . . Cai, Q. (2019). Five-long non-coding RNA risk score system for the effective prediction of gastric cancer patient survival. Oncol Lett, 17(5), 4474-4486. doi:10.3892/ol.2019.10124

Jones, G. T., Bown, M. J., Gretarsdottir, S., Romaine, S. P., Helgadottir, A., Yu, G., . . van Rij, A. M. (2013). A sequence variant associated with sortilin-1 (SORT1) on 1p13.3 is independently associated with abdominal aortic aneurysm. Hum Mol Genet, 22(14), 2941-2947. doi:10.1093/hmg/ddt141 
455

456

457

458

459

460

461

462

463

464

465

466

467

468

469

470

471

472

473

474

475

476

477

478

479

480

481

482

483

484

485

486

487

488

489

490

491

492

493

494

495

496

497

498

499

500

501

502

503

504

505

506

507

508

509

510

Jones, G. T., Tromp, G., Kuivaniemi, H., Gretarsdottir, S., Baas, A. F., Giusti, B., . . Bown, M. J. (2017). MetaAnalysis of Genome-Wide Association Studies for Abdominal Aortic Aneurysm Identifies Four New Disease-Specific Risk Loci. Circ Res, 120(2), 341-353. doi:10.1161/circresaha.116.308765

Kranjc, T., Dempsey, E., Cagney, G., Nakamura, N., Shields, D. C., \& Simpson, J. C. (2017). Functional characterisation of the YIPF protein family in mammalian cells. Histochem Cell Biol, 147(4), 439-451. doi:10.1007/s00418-016-1527-3

Langfelder, P., \& Horvath, S. (2008). WGCNA: an R package for weighted correlation network analysis. $B M C$ Bioinformatics, 9, 559. doi:10.1186/1471-2105-9-559

Lareyre, F., Clement, M., Raffort, J., Pohlod, S., Patel, M., Esposito, B., . . Mallat, Z. (2017). TGFbeta (Transforming Growth Factor-beta) Blockade Induces a Human-Like Disease in a Nondissecting Mouse Model of Abdominal Aortic Aneurysm. Arterioscler Thromb Vasc Biol, 37(11), 2171-2181. doi:10.1161/atvbaha.117.309999

Lenk, G. M., Tromp, G., Weinsheimer, S., Gatalica, Z., Berguer, R., \& Kuivaniemi, H. (2007). Whole genome expression profiling reveals a significant role for immune function in human abdominal aortic aneurysms. BMC Genomics, 8, 237. doi:10.1186/1471-2164-8-237

Li, J., Pan, C., Zhang, S., Spin, J. M., Deng, A., Leung, L. L. K., . . Snyder, M. (2018). Decoding the Genomics of Abdominal Aortic Aneurysm. Cell, 174(6), 1361-1372.e1310. doi:10.1016/j.cell.2018.07.021

Liston, A., Papadopoulou, A. S., Danso-Abeam, D., \& Dooley, J. (2012). MicroRNA-29 in the adaptive immune system: setting the threshold. Cell Mol Life Sci, 69(21), 3533-3541. doi:10.1007/s00018-012-1124-0

Ma, X., Tao, R., Li, L., Chen, H., Liu, Z., Bai, J., . . Tao, K. (2019). Identification of a 5microRNA signature and hub miRNAmRNA interactions associated with pancreatic cancer. Oncol Rep, 41(1), 292-300. doi:10.3892/or.2018.6820

Meng, X., Yang, J., Dong, M., Zhang, K., Tu, E., Gao, Q., . . Zhang, Y. (2016). Regulatory T cells in cardiovascular diseases. Nat Rev Cardiol, 13(3), 167-179. doi:10.1038/nrcardio.2015.169

Nordon, I. M., Hinchliffe, R. J., Loftus, I. M., \& Thompson, M. M. (2011). Pathophysiology and epidemiology of abdominal aortic aneurysms. Nat Rev Cardiol, 8(2), 92-102. doi:10.1038/nrcardio.2010.180

Ono, J., \& Okada, Y. (2018). Study of MYB-NFIB chimeric gene expression, tumor angiogenesis, and proliferation in adenoid cystic carcinoma of salivary gland. Odontology, 106(3), 238-244. doi:10.1007/s10266-017-03261

Raffort, J., Lareyre, F., Clement, M., Hassen-Khodja, R., Chinetti, G., \& Mallat, Z. (2017). Monocytes and macrophages in abdominal aortic aneurysm. Nat Rev Cardiol, 14(8), 457-471. doi:10.1038/nrcardio.2017.52

Reiterer, V., Figueras-Puig, C., Le Guerroue, F., Confalonieri, S., Vecchi, M., Jalapothu, D., . . Farhan, H. (2017). The pseudophosphatase STYX targets the F-box of FBXW7 and inhibits SCFFBXW7 function. Embo $j$, 36(3), 260-273. doi:10.15252/embj.201694795

Reiterer, V., Pawlowski, K., \& Farhan, H. (2017). STYX: a versatile pseudophosphatase. Biochem Soc Trans, 45(2), 449-456. doi:10.1042/bst20160279

Sakalihasan, N., Michel, J. B., Katsargyris, A., Kuivaniemi, H., Defraigne, J. O., Nchimi, A., . . Hultgren, R. (2018). Abdominal aortic aneurysms. Nat Rev Dis Primers, 4(1), 34. doi:10.1038/s41572-018-0030-7

Senemaud, J., Caligiuri, G., Etienne, H., Delbosc, S., Michel, J. B., \& Coscas, R. (2017). Translational Relevance and Recent Advances of Animal Models of Abdominal Aortic Aneurysm. Arterioscler Thromb Vasc Biol, 37(3), 401-410. doi:10.1161/atvbaha.116.308534

Shui, J. W., Boomer, J. S., Han, J., Xu, J., Dement, G. A., Zhou, G., \& Tan, T. H. (2007). Hematopoietic progenitor kinase 1 negatively regulates $\mathrm{T}$ cell receptor signaling and T cell-mediated immune responses. Nat Immunol, 8(1), 84-91. doi:10.1038/ni1416

Spin, J. M., Hsu, M., Azuma, J., Tedesco, M. M., Deng, A., Dyer, J. S., .. Tsao, P. S. (2011). Transcriptional profiling and network analysis of the murine angiotensin II-induced abdominal aortic aneurysm. Physiol Genomics, 43(17), 993-1003. doi:10.1152/physiolgenomics.00044.2011

Trachet, B., Piersigilli, A., Fraga-Silva, R. A., Aslanidou, L., Sordet-Dessimoz, J., Astolfo, A., . . Stergiopulos, N. (2016). Ascending Aortic Aneurysm in Angiotensin II-Infused Mice: Formation, Progression, and the Role of Focal Dissections. Arterioscler Thromb Vasc Biol, 36(4), 673-681. doi:10.1161/atvbaha.116.307211

Tsuruda, T., Kato, J., Hatakeyama, K., Kojima, K., Yano, M., Yano, Y., . . Kitamura, K. (2008). Adventitial mast cells contribute to pathogenesis in the progression of abdominal aortic aneurysm. Circ Res, 102(11), 13681377. doi:10.1161/circresaha.108.173682

van Dam, S., Vosa, U., van der Graaf, A., Franke, L., \& de Magalhaes, J. P. (2018). Gene co-expression analysis for functional classification and gene-disease predictions. Brief Bioinform, 19(4), 575-592. doi:10.1093/bib/bbw139 
511 Wan, L., Huang, J., Ni, H., \& Yu, G. (2018). Screening key genes for abdominal aortic aneurysm based on gene expression omnibus dataset. BMC Cardiovasc Disord, 18(1), 34. doi:10.1186/s12872-018-0766-8

Weintraub, N. L. (2009). Understanding abdominal aortic aneurysm. $N$ Engl J Med, 361(11), 1114-1116. doi:10.1056/NEJMcibr0905244

Yodoi, K., Yamashita, T., Sasaki, N., Kasahara, K., Emoto, T., Matsumoto, T., . . . Hirata, K. (2015). Foxp3+ regulatory $\mathrm{T}$ cells play a protective role in angiotensin II-induced aortic aneurysm formation in mice. Hypertension, 65(4), 889-895. doi:10.1161/hypertensionaha.114.04934

Zhang, B., \& Horvath, S. (2005). A general framework for weighted gene co-expression network analysis. Stat Appl Genet Mol Biol, 4, Article17. doi:10.2202/1544-6115.1128

Zhang, J., Sun, J., Lindholt, J. S., Sukhova, G. K., Sinnamon, M., Stevens, R. L., . . Shi, G. P. (2011). Mast cell tryptase deficiency attenuates mouse abdominal aortic aneurysm formation. Circ Res, 108(11), 1316-1327. doi:10.1161/circresaha.111.243758

Zhou, Y., Wu, W., Lindholt, J. S., Sukhova, G. K., Libby, P., Yu, X., \& Shi, G. P. (2015). Regulatory T cells in human and angiotensin II-induced mouse abdominal aortic aneurysms. Cardiovasc Res, 107(1), 98-107. doi:10.1093/cvr/cvv119 
Figure 1

Workflow for the whole study

Differential expressed gene (DEG) identification in AAA versus normal
Co-expression analysis on AAA and normal condition (based on WGCNA)

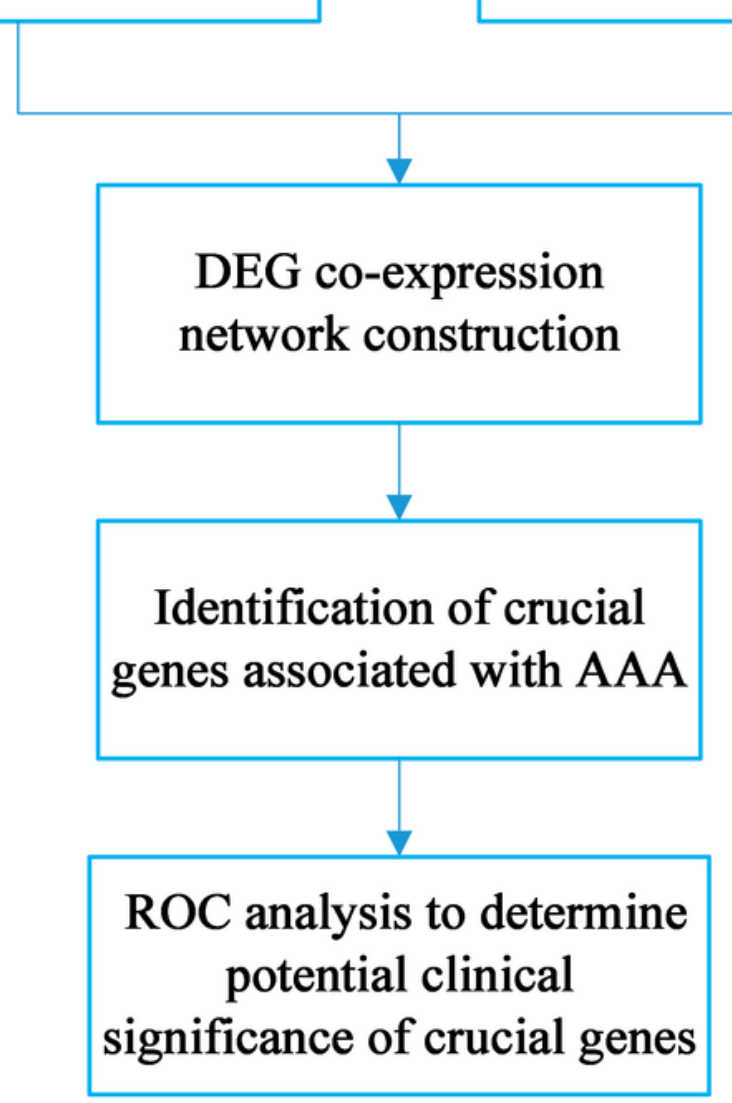

Functional modules detection

Comparison of biological function of modules between AAA and normal condition 


\section{Figure 2}

Differential expressed genes analysis.

(A) Heatmap of 143 DEGs. The diagram presents the result of a two-way hierarchical clustering of all the DEGs and samples. (B)X-axis represents -log(adj.P.Val) and Y-axis represents $\log$ FC. Magenta dots represent genes with $\log F C>0.8$ and $-\log ($ adj.P.Val) $<0.05$. Green dots represent genes with $\log F C<-0.8$ and $-\log ($ adj.P.Val) $<0.05$ (C) Black bar represents the number of down-regulated genes. Grey dots represents the number of upregulated genes.



\section{Figure 3}

WGCNA on AAA conditions.

(A) Sample clustering was conducted to detect outliers. All samples are located in the clusters and pass the cutoff thresholds. (B-C)Soft-thresholding power analysis was used to obtain the scale-free fit index of network topology. (D-E)Scale free topology when softthresholding power $\beta=5$.(F) Hierarchical cluster analysis was conducted to detect coexpression clusters with corresponding color assignments. Each color represents a module in the constructed gene co-expression network by WGCNA. (G)Heatmap depicts the Topological Overlap Matrix (TOM) of genes selected for weighted co-expression network analysis. Light color represents lower overlap and red represents higher overlap.

A
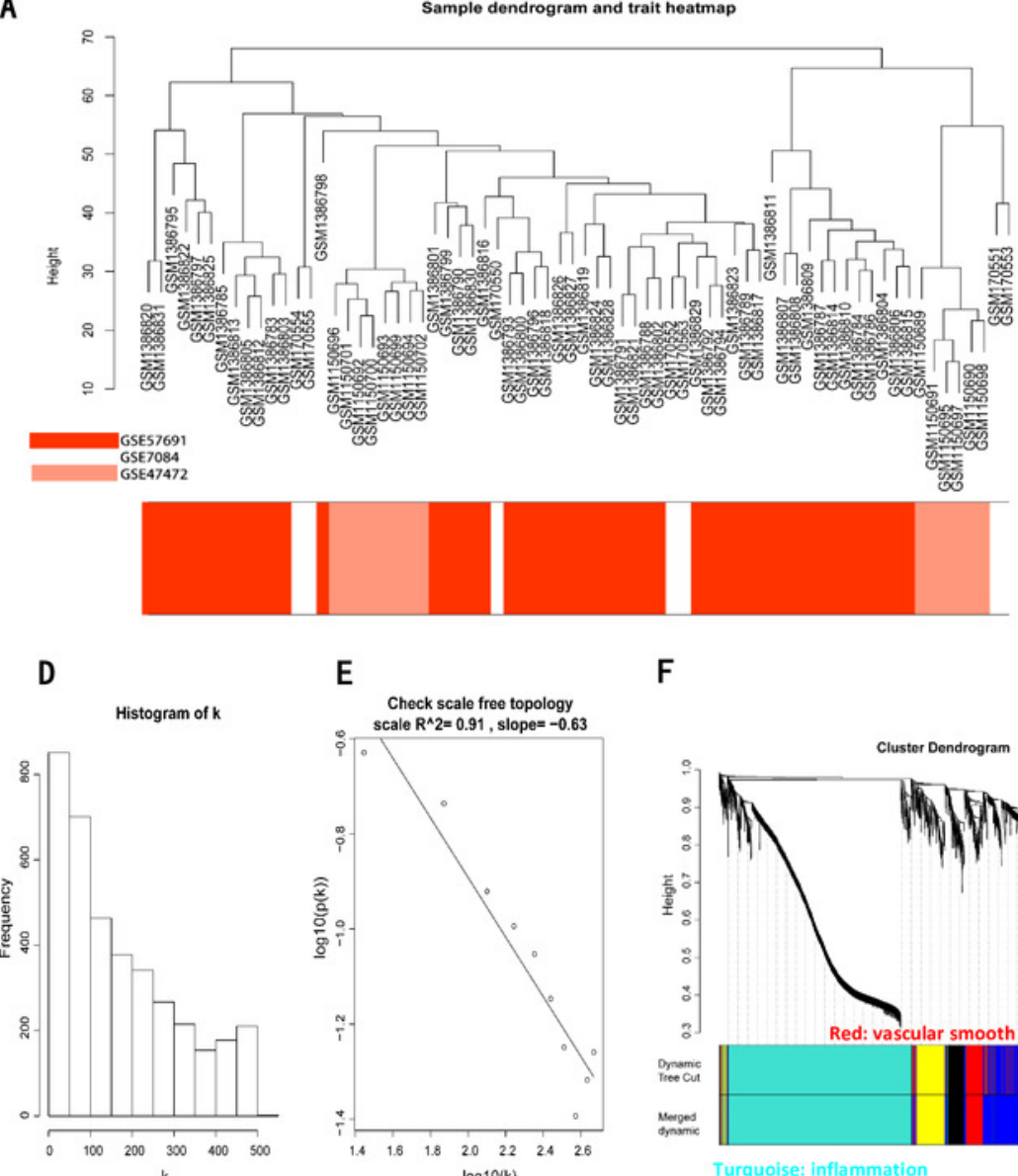

E

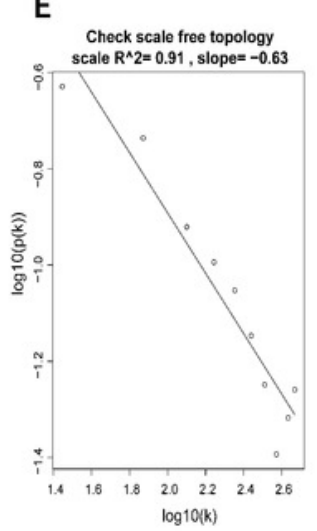

F
B

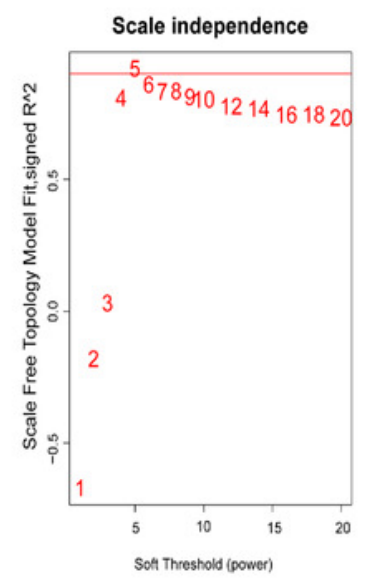

G

C
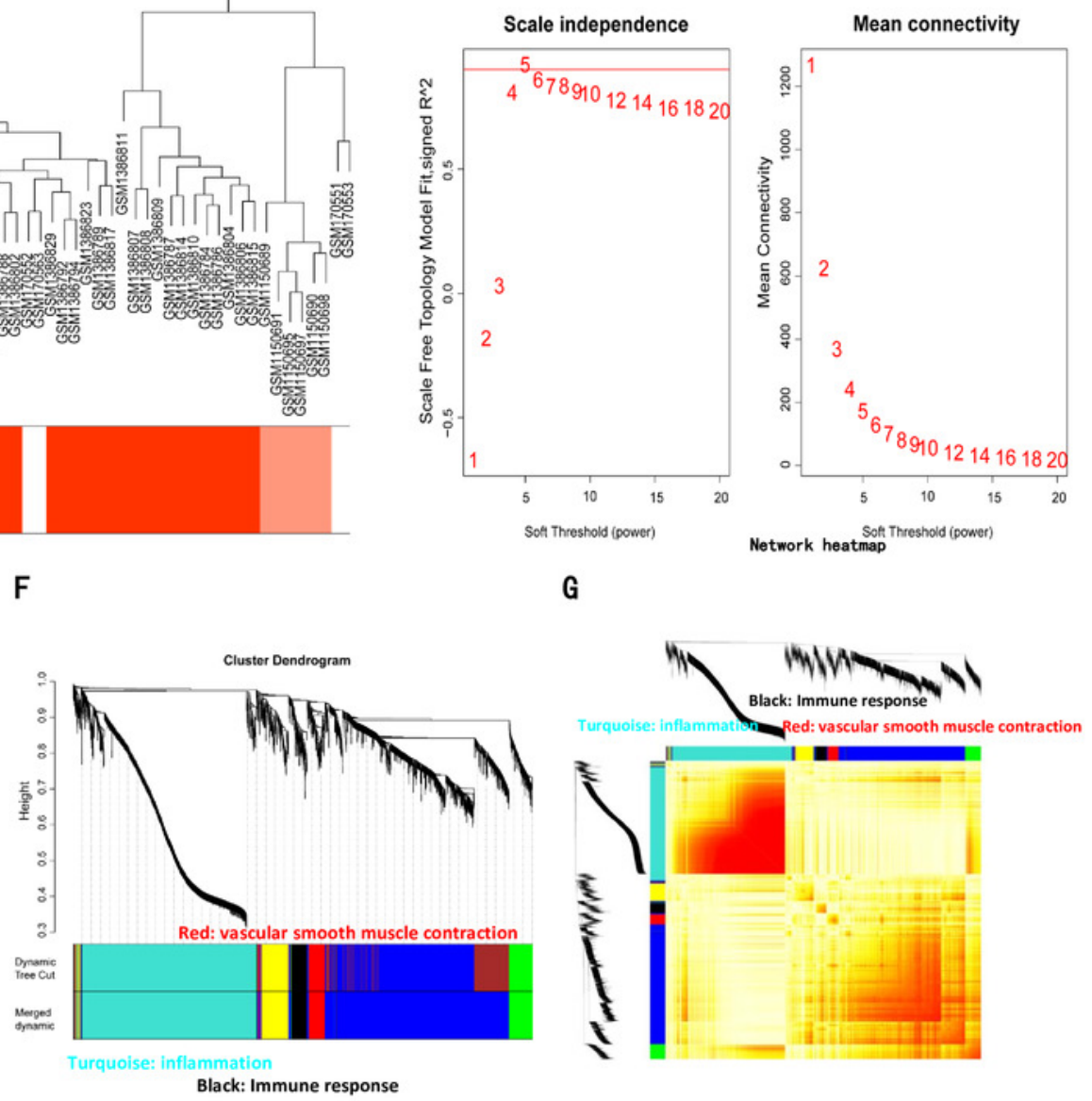
Figure 4

Significantly enriched biological processes and KEGG pathways with top10 count number of genes in 3 modules on AAA condition

The left axis represents the count number. The right axis represent the -logP 


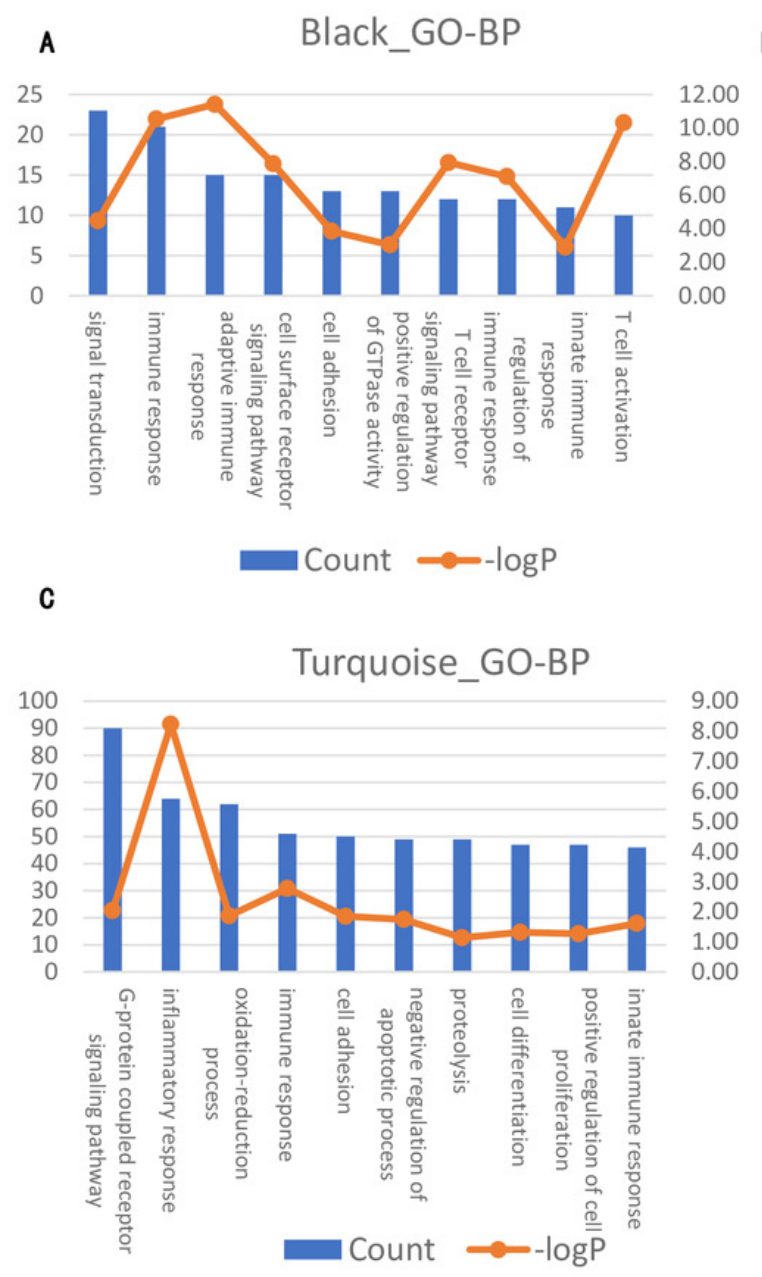

B

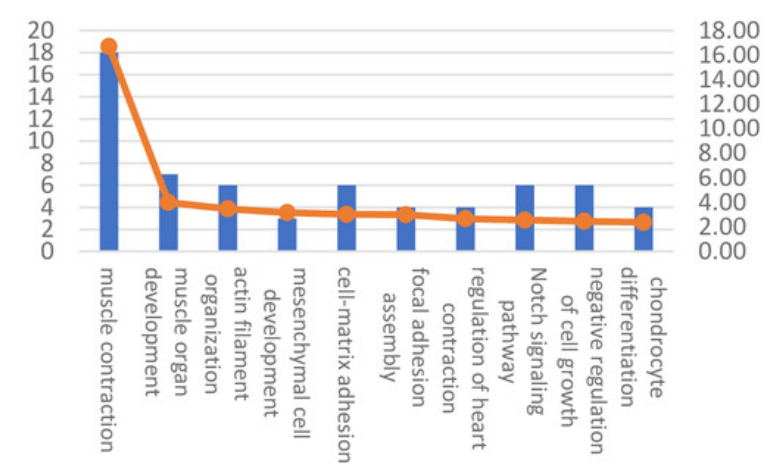

D

Count $\rightarrow-\log P$

\section{Black_KEGG}
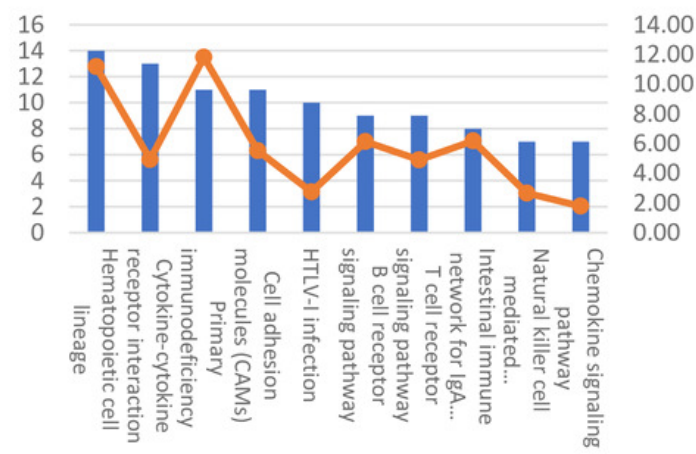

$\varpi$ Count $\multimap-\log P$

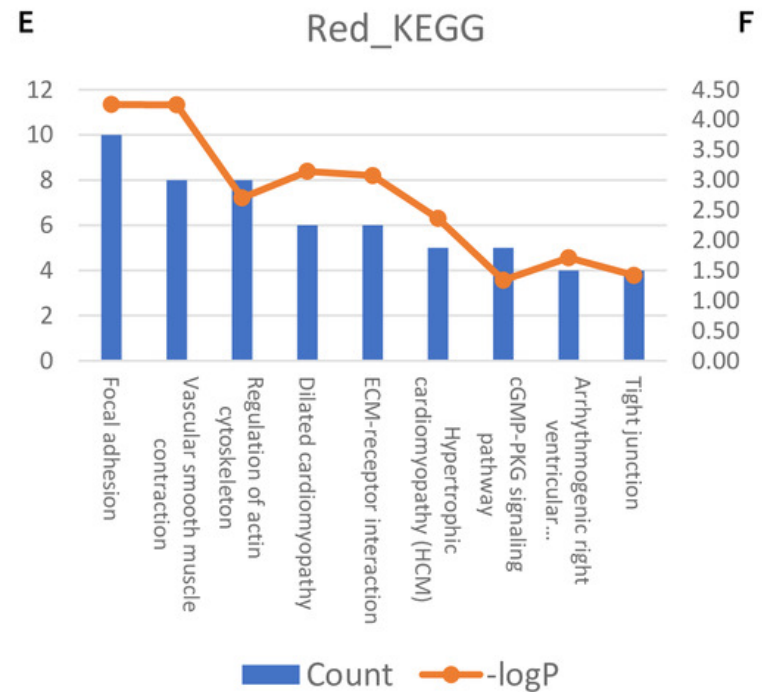

$\mathbf{F}$

Turquoise_KEGG

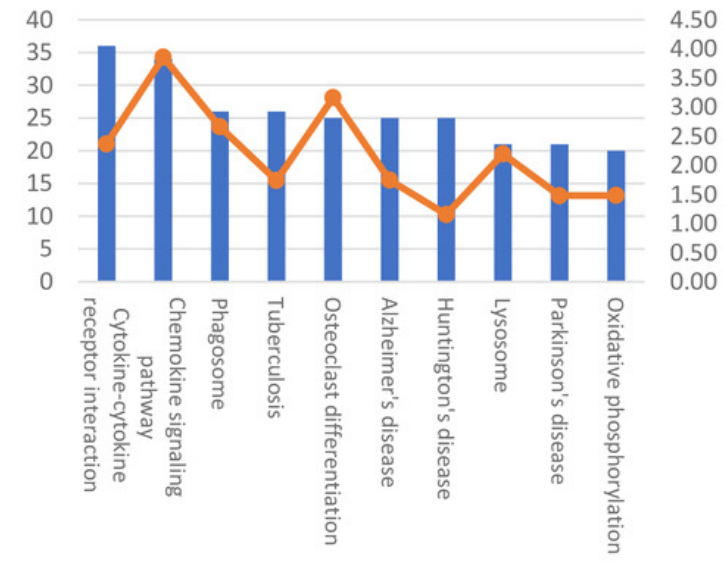

Count $\multimap-\log P$ 
Figure 5

DEG co-expression network and hub gene cluster

(A) In this DEG co-expression network, Green boxes represent down-regualted genes. Lightyellow boxes represent up-regulated genes. (B) Hub gene cluster of DEG co-expression network

A

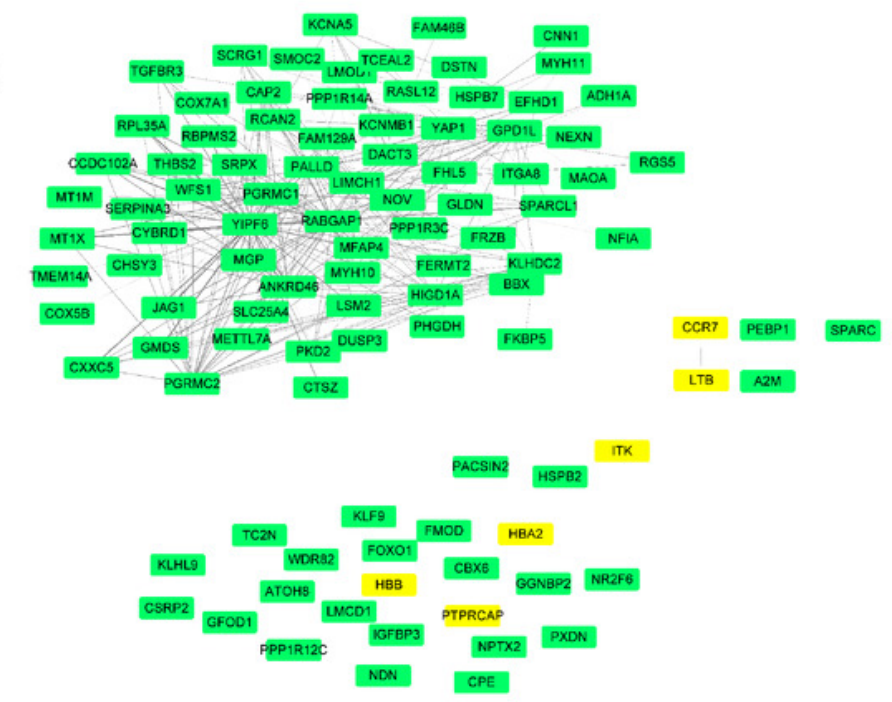

B




Figure 6

\section{ROC curves of genes with AUC exceeding 0.8}
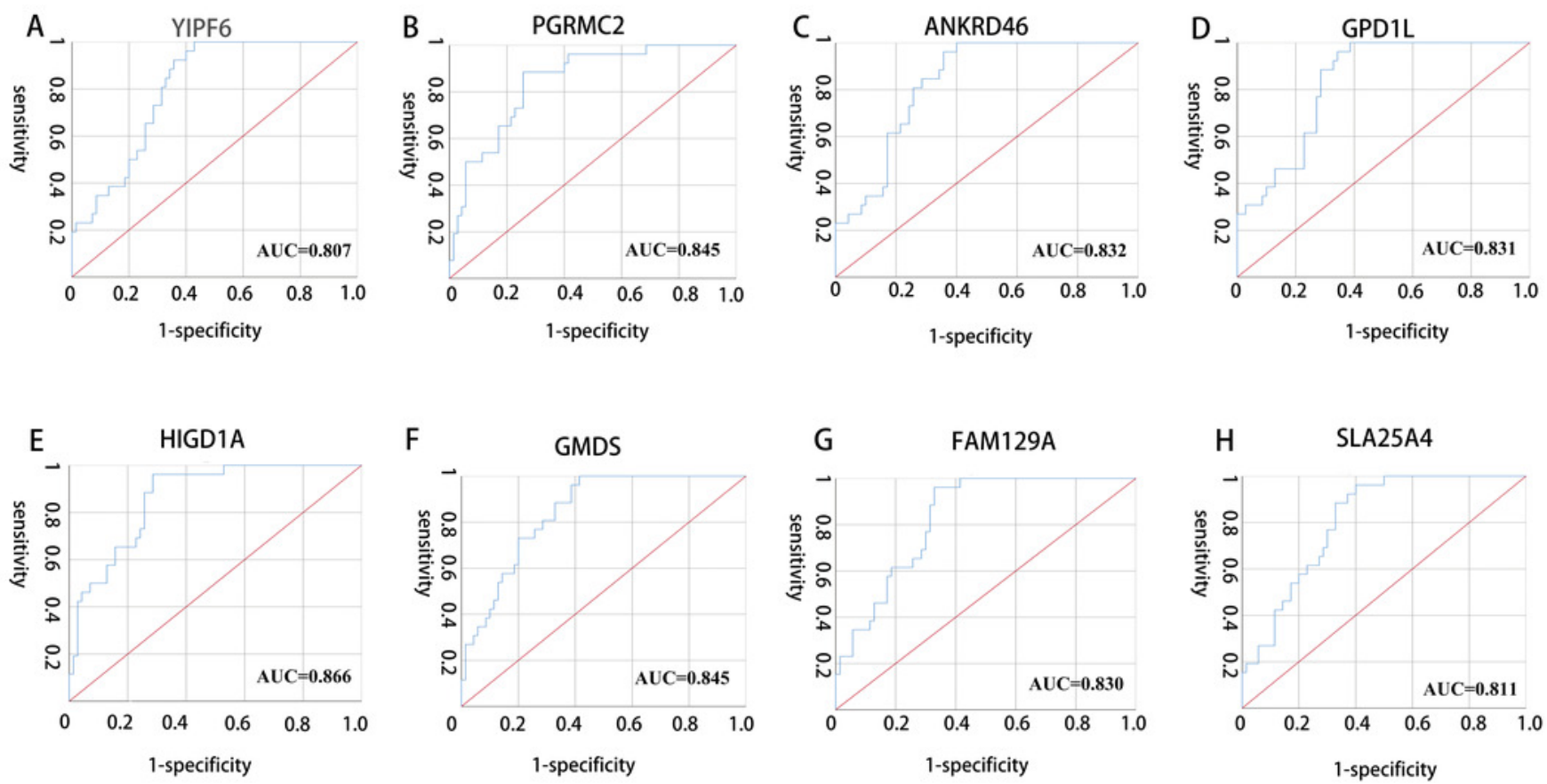
Table $\mathbf{1}$ (on next page)

Basic information of three datasets 
1 Table1 Basic information of three datasets

\begin{tabular}{cccc}
\hline Year & Series & Platform & Samples (AAA : Normal) \\
\hline 2007 & GSE7084 & $\begin{array}{c}\text { GPL2507 Sentrix Human-6 Expression } \\
\text { BeadChip }\end{array}$ & $7: 8$ \\
2013 & GSE47472 & $\begin{array}{c}\text { GPL10558 Illumina HumanHT-12 V4.0 } \\
\text { expression beadchip }\end{array}$ & $14: 8$ \\
2015 & GSE57691 & $\begin{array}{c}\text { GPL10558 Illumina HumanHT-12 V4.0 } \\
\text { expression beadchip }\end{array}$ & $49: 10$ \\
\hline
\end{tabular}

2 
Table 2 (on next page)

Comparison between AAA and normal co-expression network 
Table 2 Comparison between AAA and normal co-expression network 2

\begin{tabular}{|c|c|c|c|c|c|c|c|c|c|}
\hline & $\begin{array}{l}\text { AAA- } \\
\text { black }\end{array}$ & $\begin{array}{l}\text { Normal- } \\
\text { darkgreen }\end{array}$ & $\begin{array}{l}\mathrm{p} \\
\text { value }\end{array}$ & $\begin{array}{l}\text { AAA- } \\
\text { turquoise }\end{array}$ & $\begin{array}{l}\text { Normal- } \\
\text { turquoise }\end{array}$ & $\begin{array}{l}\mathrm{p} \\
\text { value }\end{array}$ & $\begin{array}{l}\text { AAA- } \\
\text { red }\end{array}$ & $\begin{array}{l}\text { Normal- } \\
\text { brown }\end{array}$ & $\begin{array}{l}\mathrm{P} \\
\text { value }\end{array}$ \\
\hline & $\mathrm{n}=50$ & $\mathrm{n}=30$ & & $\mathrm{n}=153$ & $\mathrm{n}=155$ & & $n=26$ & $\mathrm{n}=49$ & \\
\hline Related genes \% & $72.0 \%$ & $46.7 \%$ & 0.023 & $52.3 \%$ & $40.6 \%$ & 0.041 & $50.0 \%$ & $14.3 \%$ & 0.001 \\
\hline Other genes\% & $28.0 \%$ & $53.3 \%$ & & $47.7 \%$ & $59.4 \%$ & & $50.0 \%$ & $85.7 \%$ & \\
\hline
\end{tabular}


Table 3 (on next page)

Genes in the hub gene cluster of DEG co-expression network 
1 Table 3 Genes in the hub gene cluster of DEG co-expression network

\begin{tabular}{llll}
\hline & Gene ID & Gene symbol & Official full name \\
\hline 1 & 286451 & YIPF6 & Yip1 domain family member 6 \\
2 & 23637 & RABGAP1 & RAB GTPase activating protein 1 \\
3 & 157567 & ANKRD46 & ankyrin repeat domain 46 \\
4 & 23171 & GPD1L & glycerol-3-phosphate dehydrogenase 1 like \\
5 & 10424 & PGRMC2 & progesterone receptor membrane component 2 \\
6 & 25994 & HIGD1A & HIG1 hypoxia inducible domain family member 1A \\
7 & 2762 & GMDS & GDP-mannose 4,6-dehydratase \\
8 & 4256 & MGP & matrix Gla protein \\
9 & 291 & SLC25A4 & solute carrier family 25 member 4 \\
10 & 116496 & FAM129A & niban apoptosis regulator 1 \\
\hline
\end{tabular}

2 


\section{Table 4 (on next page)}

The area under the curve (AUC) of 10 genes in the cluster detected by MCODE 
1 Table 4 The area under the curve (AUC) of 10 genes in the cluster detected by MCODE

\begin{tabular}{lll}
\hline Gene symbol & AUC & P value \\
\hline YIPF6 & 0.807 & $<0.001$ \\
RABGAP1 & 0.797 & $<0.001$ \\
PGRMC2 & 0.845 & $<0.001$ \\
ANKRD46 & 0.832 & $<0.001$ \\
GPD1L & 0.831 & $<0.001$ \\
HIGD1A & 0.866 & $<0.001$ \\
GMDS & 0.845 & $<0.001$ \\
MGP & 0.742 & $<0.001$ \\
FAM129A & 0.830 & $<0.001$ \\
SLC25A4 & 0.811 & $<0.001$ \\
\hline
\end{tabular}

2

3

4

5 